\title{
Clinical outcomes of endovascular treatment for ruptured thoracic aortic disease
}

\author{
Jong Hyun Choi ${ }^{1,}$, Sang-Pil Kim ${ }^{2,}$, Han Cheol Lee ${ }^{3}$, Tae Sik Park ${ }^{3}$, Jong Ha Park ${ }^{3}$, Bo Won Kim³, \\ Jinhee $\mathrm{Ahn}^{3}$, Jin Sup Park ${ }^{3}$, Hye Won Lee ${ }^{3}$, Jun-Hyok Oh³ , Jung Hyun Choi ${ }^{3}$, Kwang Soo Cha ${ }^{3}$, and \\ Taek Jong Hong ${ }^{3}$
}

${ }^{1}$ Division of Cardiology, Department of Internal Medicine, Busan Veterans Hospital, Busan; ${ }^{2}$ Department of Thoracic Surgery, ${ }^{3}$ Division of Cardiology, Department of Internal Medicine, Medical Research Institute, Pusan National University Hospital, Busan, Korea

Received: March 7, 2019

Revised : May 8, 2019

Accepted: July 15, 2019

\section{Correspondence to}

Han Cheol Lee, M.D.

Division of Cardiology,

Department of Internal

Medicine, Pusan National

University Hospital, 179

Gudeok-ro, Seo-gu, Busan

49241, Korea

Tel.: +82-51-240-7217

Fax: +82-51-240-7795

E-mail: glaraone@hanmail.net

https://orcid.org/0000-0002-

7236-4204

*'These authors contributed equally to this work.
Background/Aims: Untreated rupture of the thoracic aorta is associated with a high mortality rate. We aimed to review the clinical results of endovascular treatment for ruptured thoracic aortic disease.

Methods: We retrospectively reviewed data on 37 patients (mean age, 67.0 \pm 15.18 years) treated for ruptured thoracic aortic disease from January 2005 to May 2016. The median follow-up duration was 308 days (interquartile range, 61 to $1,036.5$ ). The primary end-point of the study was the composite of death, secondary intervention, endoleak, and major stroke/paraplegia after endovascular treatment.

Results: The etiologies of ruptured thoracic aortic disease were aortic dissection (n $=11,29.7 \%)$, intramural hematoma $(\mathrm{n}=7,18.9 \%)$, thoracic aortic aneurysm $(\mathrm{n}=14$, $37.8 \%)$, and traumatic aortic transection $(\mathrm{n}=5,13.5 \%)$. Three patients died within 24 hours of thoracic endovascular aortic repair, and one showed type I endoleak. The technical success rate was $89.2 \%$ (33/37). The in-hospital mortality rate was 13.5\% (5/37); no deaths occurred during follow-up. The composite outcome rate during follow-up was $37.8 \%$ (14/37), comprising death $(n=5,13.5 \%)$, secondary intervention $(n=5,13.5 \%)$, endoleak $(n=5,13.5 \%)$, and major stroke/paraplegia $(n=3$, 8.1\%). Left subclavian artery revascularization and proximal landing zone were not associated with the composite outcome. Low mean arterial pressure (MAP; $\leq$ $60 \mathrm{mmHg}$, [hazard ratio, 13.018; 95\% confidence interval, 2.435 to $69.583, p=0.003$ ]) was the most significant predictor and high transfusion requirement in the first 24 hours was associated with event-free survival $(\log \operatorname{rank} p=0.018)$.

Conclusions: Endovascular treatment achieves high technical success rates and acceptable clinical outcome. High transfusion volume and low MAP were associated with poor clinical outcomes.

Keywords: Aorta; Aortic rupture; Aneurysm, dissecting; Aortic aneurysm, thoracic; Stents

\section{INTRODUCTION}

The mortality rate of untreated ruptured thoracic aortic disease can be as high as $54 \%$ and $76 \%$ at 6 hours and 24 hours, respectively, after the initial rupture event [1].
Traditionally, these patients were treated with open surgical repair, but thoracic endovascular repair (TEVAR) has emerged as an alternative treatment for ruptured thoracic aortic disease. Jonker et al. [2] reported that TEVAR showed superior clinical outcomes over 
open repair for ruptured thoracic aortic aneurysms (composite death, stroke, or permanent paraplegia, 22\% vs. $36 \%$, respectively). However, data regarding emergency TEVAR for ruptured thoracic aortic disease is limited [3-6]. This study aimed to determine the clinical outcomes of emergent TEVAR for ruptured thoracic aortic disease and to identify the prognostic factors.

\section{METHODS}

This retrospective study included 37 patients treated for ruptured thoracic aortic disease from January 2005 to May 2016 at Pusan National University Hospital, South Korea. Ruptured thoracic aortic disease was confirmed by contrast-enhanced computed tomography (CT) before the procedure. The study's endpoint was a composite outcome consisting of death, secondary intervention, endoleak, and major stroke/paraplegia after TEVAR. We also evaluated the prognostic factors influencing the composite outcome. The study was reviewed and approved by the Institutional Review Board of Pusan National University Hospital (1890-006-070). Written informed consent by the patients was waived due to a retrospective nature of our study.

\section{Patient selection}

The treatment plan was decided by a multidisciplinary team of cardiovascular surgeons, interventionalists, cardiologists, and radiologists after considering the patients' comorbidities, life expectancy and location, and thoracic aorta anatomy. Emergent TEVAR was performed for thoracic aortic rupture due to aortic dissection, intramural hematoma, thoracic aortic aneurysm, and traumatic aortic transection. The procedure was performed using the chimney technique or partial left subclavian artery (LSCA) coverage by the main stent graft if it was involved in the aortic pathology or if the patients' vital signs were unstable. If the patients' vital signs were stable, TEVAR was performed after left carotid artery to LSCA bypass or right carotid to left carotid to LSCA bypass.

\section{Procedures}

TEVAR was performed under general or local anesthesia based on the patients' condition. The procedures were performed in the hybrid operating room, and access was obtained through the femoral artery by surgical cutdown ( $n=18,48.6 \%)$ or two perclose devices $(n=19,51.4 \%)$. Ten patients (27\%) underwent LSCA revascularization to ensure sufficient proximal landing zone. Aortography was performed with a 5-F pigtail catheter. SEAL thoracic aortic stent grafts (S\&G Biotech, Seongnam, Korea) were used in all patients.

We defined technical success as accurate deployment of the stent graft and exclusion of the rupture site without type I or III endoleaks or mortality within 24 hours.

\section{Clinical and imaging follow-up}

The patients were regularly followed up at 1, 3, 6, and 12 months, and every 3 months thereafter, at the outpatient clinics to determine their clinical status and check for complications. CT scans were performed at 1, 3, 6, and 12 months, and annually thereafter, to identify endoleaks and changes to the thoracic aorta.

\section{Statistical analysis}

All data were expressed as mean \pm standard deviation or frequency and percentage. Kaplan-Meier survival models were used to compare outcomes according to pathology, need for massive transfusion, and treatment approach. A Cox proportional hazards regression model was created to identify possible predictors of the composite outcome during follow up. All possible predictors were entered into a multivariable cox regression model (stepwise backward elimination).

Statistical analyses were performed using the software package SPSS version 11.o (SPSS Inc., Chicago, IL, USA). A $p<0.05$ was considered statistically significant.

\section{RESULTS}

The baseline characteristics and the procedural characteristics of the patients are summarized in Tables 1 and 2. The mean age was $67.0 \pm 15.2$ years (range, 25 to 84 ), and 23 patients were men (62.2\%). Most patients had stable vital signs on admission; however, four patients had a low mean arterial pressure (MAP; < $60 \mathrm{mmHg}$ ). Hypertension was the most common risk factor (73.0\%).

Clinical outcomes are summarized in Table 3. The patients were divided into four groups according to the 
Table 1. Baseline patient characteristics $(n=37)$

\begin{tabular}{lc}
\hline Characteristic & Value \\
\hline Age, $\mathrm{yr}$ & $67.1 \pm 15.3$ \\
Men & $23(62.2)$ \\
BMI, $\mathrm{kg} / \mathrm{m}^{2}$ & $23.6 \pm 3.5$ \\
SBP on admission, $\mathrm{mmHg}$ & $123.5 \pm 40.3$ \\
\hline DBP on admission, $\mathrm{mmHg}$ & $74.5 \pm 22.4$ \\
MAP on admission, mmHg & $90.8 \pm 27.9$ \\
Biomarkers & \\
\hline WBC, $/ \mu \mathrm{L}$ & $11,056.8 \pm 5.310 .1$ \\
\hline Hemoglobin, g/dL & $11.4 \pm 2.2$ \\
\hline Creatinine, mg/dL & $1.3 \pm 0.8$ \\
\hline C-reactive protein, mg/dL & $3.2 \pm 4.2$ \\
Risk factors & \\
\hline Hypertension & $27(73.0)$ \\
\hline Diabetes & $5(13.5)$ \\
\hline Smoking & $11(29.7)$ \\
\hline Hyperlipidemia & $1(2.7)$ \\
Coronary artery disease & $3(8.1)$ \\
\hline Previous MI & $1(2.7)$ \\
\hline Cerebrovascular accident & $4(10.8)$ \\
\hline
\end{tabular}

Values are presented as mean $\pm \mathrm{SD}$ or number (\%). BMI, body mass index; SBP, systolic blood pressure; DBP, diastolic blood pressure; MAP, mean arterial pressure; WBC, white blood cell count; MI, myocardial infarction.

etiology of thoracic aortic rupture: aortic dissection $(\mathrm{n}=$ $11,29.7 \%)$, intramural hematoma $(n=7,18.9 \%)$, thoracic aortic aneurysm $(n=14,37.8 \%)$, and traumatic aortic transection $(n=5,13.5 \%)$. Three patients died within 24 hours of TEVAR and one showed type I endoleak. The technical success rate was $89.2 \%(\mathrm{n}=33)$.

The in-hospital mortality rate was $13.5 \%(\mathrm{n}=5)$, and no deaths occurred during follow-up. The composite outcome rate during follow-up was 37.8\% ( $\mathrm{n}=14)$, comprising death $(\mathrm{n}=5,13.5 \%)$, secondary intervention $(\mathrm{n}=5$, $13.5 \%)$, endoleak $(n=5,13.5 \%)$, and major stroke/paraplegia $(n=3,8.1 \%)$. In the intramural hematoma group, the composite outcome occurred in only one patient (14.3\%) during follow-up compared to seven patients (50\%) in the thoracic aortic aneurysm group, but the difference was not statistically significant. The Kaplan-Meier analysis revealed that event-free survival did not show a statistically significant difference between the different eti-
Table 2. Procedural characteristics $(n=37)$

\begin{tabular}{lc}
\hline Characteristic & Value \\
\hline No. of stent grafts & $2 \pm 1.3$ \\
Total length of stent graft, mm & $226.1 \pm 110.1$ \\
\hline Stent graft diameter, mm & $39.9 \pm 5.2$ \\
LSCA revascularization & $10(27)$ \\
$\quad$ Chimney technique & $7(18.9)$ \\
$\quad$ Left carotid to LSCA bypass & $1(2.7)$ \\
Right carotid to carotid to LSCA bypass & $2(5.4)$ \\
Proximal landing zone & \\
\hline 1 & $4(10.8)$ \\
\hline 2 & $15(40.5)$ \\
3 & $13(35.1)$ \\
\hline 4 & $5(13.5)$ \\
Perclose device closure & $19(51.4)$ \\
Surgical cutdown & $17(48.6)$ \\
\hline 24 Hours pRBC requirement, unit & $5.81 \pm 7.96$ \\
\hline
\end{tabular}

Values are presented as mean \pm SD or number (\%). LSCA, left subclavian artery; pRBC, pack red blood cell.

ologies ( $\log \operatorname{rank} p=0.566$ ) (Fig. 1), but a high transfusion requirement in the first 24 hours was associated with event-free survival (log rank $p=0.018$ ) (Fig. 2).

We also conducted univariate Cox regression analysis to determine prognostic factors influencing the composite outcome. A low MAP ( $\leq 60 \mathrm{mmHg}$ ) was significantly associated with the composite outcome (hazard ratio [HR], 11.276; 95\% confidence interval [CI], 2.934 to $43.334, p<0.001)$, and occurred in four patients. Among these, three patients died, and one developed endoleak. High transfusion volumes ( $\geq 5$ units) of packed red blood cells during the first 24 hours was also associated with the composite outcome (HR, 3.435; 95\% CI, 1.146 to 10.300; $p=0.028$ ). However, a low MAP was the only independent predictors associated with the composite outcome in multivariate cox regression analysis (HR, 13.018; $95 \%$ CI, 2.435 to $69.583, p=0.003$ ).

Initial biomarkers (white blood cell, hemoglobin, creatinine and C-reactive protein on admission), cardiovascular risk factors, and comorbidities were not significant predictors of the composite outcome. The proximal landing zone of the stent graft was also not a significant factor; the zone 1 to 2 group did not show an increase in the composite outcome compared with 
Table 3. Clinical outcomes based on ruptured thoracic aortic disease

\begin{tabular}{|c|c|c|c|c|c|c|}
\hline Variable & Total $(\mathrm{n}=37)$ & $\mathrm{AD}(\mathrm{n}=11)$ & $\mathrm{IMH}(\mathrm{n}=7)$ & $\operatorname{TAA}(\mathrm{n}=14)$ & $\operatorname{TAT}(\mathrm{n}=5)$ & $p$ value \\
\hline Hospital length of stay, day & $21.1 \pm 19.1$ & $22.5 \pm 20.4$ & $24.9 \pm 29.8$ & $17.9 \pm 14.6$ & $21.6 \pm 12.5$ & 0.744 \\
\hline Technical success & $33(89.2)$ & $9(81.8)$ & $6(85 \cdot 7)$ & $13(92.9)$ & $5(100)$ & 0.680 \\
\hline Total length of stent graft, $\mathrm{mm}$ & $226.1 \pm 110.1$ & $228.2 \pm 102.6$ & $242.1 \pm 78.2$ & $252.6 \pm 132.9$ & $125 \cdot 0 \pm 23 \cdot 9$ & 0.022 \\
\hline Peak CRP & $18.9 \pm 7.4$ & $16.9 \pm 4.8$ & $24.6 \pm 10.2$ & $19.1 \pm 7.5$ & $15.0 \pm 4.0$ & 0.476 \\
\hline \multicolumn{7}{|l|}{ In-hospital clinical outcome } \\
\hline Composite outcome & $10(27.0)$ & $3(27 \cdot 3)$ & $1(14 \cdot 3)$ & $4(28.6)$ & $2(40.0)$ & 0.796 \\
\hline Death (all aorta death) & $5(13 \cdot 5)$ & $1(9.1)$ & $1(14 \cdot 3)$ & $2(14 \cdot 3)$ & $1(20)$ & 0.945 \\
\hline Secondary intervention & $1(2.7)$ & 0 & o & 0 & $1(20.0)$ & 0.087 \\
\hline Major stroke/paraplegia & $3(8.1)$ & $1(9.1)$ & $\circ(0.0)$ & $1(7.1)$ & $1(20.0)$ & 0.660 \\
\hline Secondary endoleak & $2(5 \cdot 4)$ & $1(9.1)$ & $\circ(0.0)$ & $1(7.1)$ & 0 & 0.787 \\
\hline \multicolumn{7}{|l|}{ Follow-up clinical outcome } \\
\hline Composite outcome & $14(37.8)$ & $4(36.4)$ & $1(14 \cdot 3)$ & $7(50.0)$ & $2(40.0)$ & 0.466 \\
\hline Death (all aorta death) & $5(13 \cdot 5)$ & $1(9.1)$ & $1(14 \cdot 3)$ & $2(14 \cdot 3)$ & $1(20.0)$ & 0.945 \\
\hline Secondary intervention & $5(13.5)$ & o & o & $4(28.6)$ & $1(20.0)$ & 0.127 \\
\hline Major stroke/paraplegia & $3(8.1)$ & $1(9.1)$ & $\mathrm{O}$ & $1(7 \cdot 1)$ & $1(20.0)$ & 0.660 \\
\hline Secondary endoleak & $5(13 \cdot 5)$ & $2(18.2)$ & o & $3(21.4)$ & o & 0.418 \\
\hline
\end{tabular}

Values are presented as mean \pm SD or number (\%).

$\mathrm{AD}$, aortic dissection; IMH, intramural hematoma; TAA, thoracic aortic aneurysm; TAT, traumatic aortic transection; CRP, C-reactive protein.

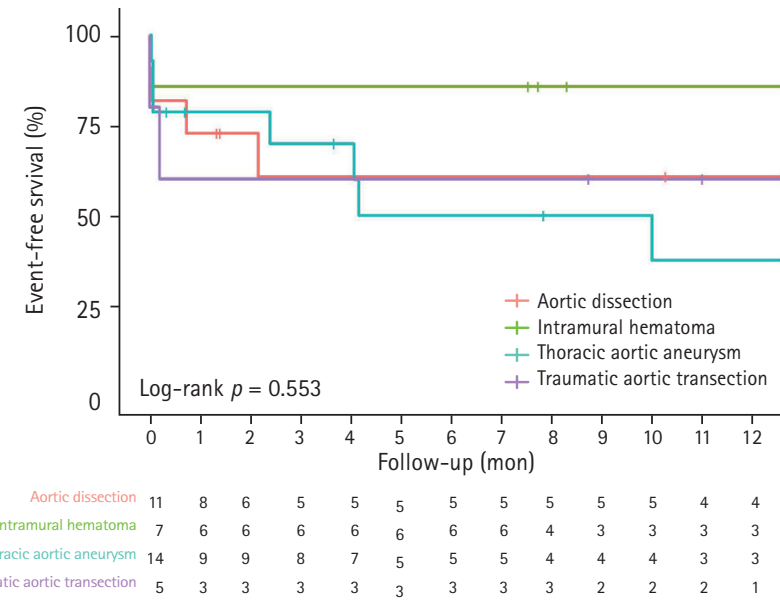

Figure 1. Kaplan-Meier analysis of event-free survival in patients. The etiologies of thoracic aortic rupture included aortic dissection $(n=11)$, intramural hematoma $(n=7)$, thoracic aortic aneurysm $(n=14)$, and traumatic aortic transection $(n=5)$. No statistically significant differences in eventfree survival between the diseases was observed $(\log \operatorname{rank} p=$ $0.566)$.

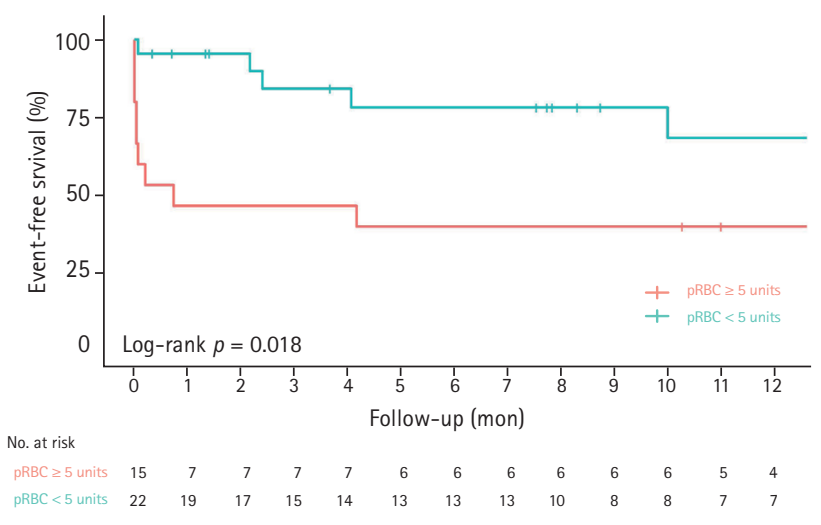

Figure 2. Kaplan-Meier analysis of event-free survival in patients with high (packed red blood cells $[\mathrm{pRBCs}] \geq 5$ units, $\mathrm{n}=22$ ), and low transfusion requirements ( $\mathrm{pRBCs}<5$ units, $\mathrm{n}=15$ ) in the first 24 hours. A high transfusion requirement in the first 24 hours was associated with the composite outcome $(\log \operatorname{rank} p=0.018)$. 
Table 4. Predictors of the composite outcome

\begin{tabular}{|c|c|c|c|c|c|c|}
\hline \multirow{2}{*}{ Variable } & \multicolumn{3}{|c|}{ Univariate analysis } & \multicolumn{3}{|c|}{ Multivariate analysis ${ }^{\mathrm{a}}$} \\
\hline & HR & $95 \%$ CI & $p$ value & HR & $95 \% \mathrm{CI}$ & $p$ value \\
\hline Low MAP, $\leq 60 \mathrm{mmHg}$ & 11.276 & $2.934-43.334$ & $<0.001$ & 13.018 & $2.435-69.583$ & 0.003 \\
\hline 24 Hours pRBC, $\geq 5$ units & 3.435 & $1.146-10.300$ & 0.028 & 3.153 & $0.838-11.864$ & 0.089 \\
\hline Diabetes & 1.577 & $0.438-5.681$ & 0.486 & 3.864 & $0.886-16.857$ & 0.072 \\
\hline Perclose device closure & 1.127 & $0.392-3.239$ & 0.825 & 2.962 & $0.852-10.299$ & 0.088 \\
\hline Age, yr & 0.993 & $0.961-1.026$ & 0.669 & - & - & - \\
\hline WBC & 1.000 & $1.000-1.000$ & 0.195 & - & - & - \\
\hline Hemoglobin & 1.042 & $0.819-1.325$ & 0.740 & - & - & - \\
\hline Smoking & 0.918 & $0.286-2.924$ & 0.881 & - & - & - \\
\hline Creatinine $>1.5$ & 1.150 & $0.320-4.123$ & 0.831 & - & - & - \\
\hline Peak CRP after TEVAR & 0.984 & $0.898-1.079$ & 0.734 & - & - & - \\
\hline Total length of stent graft, mm & 0.999 & $0.994-1.005$ & 0.832 & - & - & - \\
\hline Left subclavian artery revascularization & 0.967 & $0.303-3.087$ & 0.955 & - & - & - \\
\hline Proximal landing zone 1, 2 (vs. 3,4$)$ & 1.261 & $0.437-3.639$ & 0.668 & - & - & - \\
\hline Traumatic rupture (vs. non-traumatic) & 1.111 & $0.248-4.968$ & 0.890 & - & - & - \\
\hline
\end{tabular}

HR, hazard ratio; CI, confidence interval; MAP, mean arterial pressure; pRBC, packed red blood cell; WBC, white blood cell count; CRP, C-reactive protein; TEVAR, thoracic endovascular aortic repair.

${ }^{\mathrm{a}} \mathrm{HRs}$ (95\% CI) were adjusted for all listed variables in the table (stepwise backward elimination).

the zone 3 to 4 group, not involving the aortic arch (HR, 1.261; 95\% CI, 0.437 to 3.639). The LSCA was selectively revascularized in 10 patients (stent insertion using the chimney technique in seven, and bypass in three) and was not associated with the composite outcome (HR, 0.569 ; $95 \%$ CI, 0.303 to 3.087 ; $p=0.955$ ). The Kaplan-Meier analysis revealed that event-free survival did not show a statistical significance between the traumatic aortic transection and other groups ( $\log \operatorname{rank} p=0.889)$.

In our study, surgical cutdown or the perclose device were utilized for femoral artery access. According to the Kaplan-Meier analysis, no significant difference in event-free survival was found between the perclose device and surgical cutdown groups. C-reactive protein (CRP) level was elevated in all patients after TEVAR (mean peak, $18.93 \pm 7.37 \mathrm{mg} / \mathrm{dL}$ ). However, the high CRP level was not associated with the composite outcome in univariate Cox regression analysis (Table 4).

\section{DISCUSSION}

Ruptured thoracic aortic disease is life-threatening and is associated with a high mortality rate. Open surgery with aneurysm resection and interposition of a prosthetic graft has been the main treatment. However, open surgery for thoracic aortic rupture is associated with high mortality rates, approaching $33 \%$ to $45 \%[7,8]$. Thus, TEVAR has emerged as an alternative to open surgery [2-6]. Jonker et al. [2] reported that TEVAR for ruptured thoracic aortic aneurysms reduced death, stroke, and paraplegia, compared with open surgery (21.7\% vs. $36.2 \%$, respectively; $p=0.044$ ). In our study, the in-hospital mortality rate was $13.5 \%$, and the composite outcome rate during follow-up was $37.8 \%$. Scheinert et al. [5] reported that early mortality rate of the TEVAR for ruptured descending aortic diseases was 9.3\% $(n=3 / 31)$ and early morbidity rate was $22.6 \%$ (renal failure requiring hemodialysis $\mathrm{n}=5$, ischemic stroke $\mathrm{n}=2$ ). According to a meta-analysis of open versus endovascular repair study, the 30 -day mortality was $19 \%$ for patients treated with TEVAR for ruptured descending aortic diseases. The 30-day occurrence rates were myocardial infarction (3.5\%), stroke (4.1\%), and paraplegia (3.1\%). The estimated aneurysm-related survival at 3 years after TEVAR was 70.6\% [8]. The clinical outcome of our study was similar 
to previous reports [2-5,8-10].

TEVAR for ruptured traumatic transection has shown survival benefits compared with open surgery and promising results have been reported [11-13]. The RESCUE trial showed that all-cause mortality at 1 year was low at $12 \%$ [11]. The patients in that trial had non-diseased aortas and coexisting polytrauma. Thus, TEVAR was able to achieve good clinical outcomes due to reduced procedure time, transfusion requirement, and damage to the other organs. TEVAR for traumatic transection of non-diseased aortas has also shown reduced neurologic complications and endoleak in other studies [12-14]. In our study, five patients underwent TEVAR for ruptured traumatic aortic transection. Among them, one patient died in the hospital, and one experienced a major stroke. The Kaplan-Meier survival curve showed no statistical significance compared with non-traumatic aortic rupture. Hypovolemic shock due to massive bleeding before TEVAR was associated with mortality in our analysis. The patient who experienced the major stroke was a high-risk patient; he was an 82-year-old man with diabetes mellitus and chronic renal failure. The other patients completely recovered without complications and had short procedure times.

Some studies have shown an increased survival benefit from TEVAR compared with open surgery for ruptured aortic dissection. In addition, it could be performed in a short time and reduce blood loss and cardiovascular events $[9,10,15-17]$. In our study, among patients with ruptured aortic dissection, one died and one developed paraplegia. CT scans showed that the patient with paraplegia had an Adamkiewicz artery arising from the false lumen due to poor blood flow through it after TEVAR. Paraplegia after TEVAR is mainly associated with disease at the T8-Li level, high-risk patients with comorbidity, and sacrifice of the LSCA [18-20]. Yingbin et al. [21] reported that aortic dissection with an Adamkiewicz artery arising from a false lumen is associated with a high risk of paraplegia after TEVAR due to blockage of the false lumen.

Although there was no statistical significance, composite outcome during follow-up increased in the ruptured aortic aneurysm compared with ruptured intramural hematoma (composite outcome in the hospital, $28.6 \%$ vs. $14.3 \%$; composite outcome during follow-up, $50 \%$ vs. $14.3 \%$, respectively) in our study. The occurrence of endoleak was more frequent in the ruptured aortic aneurysm group compared with the intramural hematoma group. The proximal landing zone of the stent graft in the ruptured thoracic aortic aneurysm group was located near the aortic arch and was shorter and more curved than in the ruptured intramural hematoma group. The rupture sites in intramural hematomas are usually located in the straight descending thoracic aorta $[22,23]$. Therefore, a short, curved proximal landing zone might be related to endoleaks in ruptured thoracic aortic aneurysms.

The involvement of the left carotid artery and LSCA in thoracic aortic disease is associated with poor clinical outcomes after TEVAR $[10,24]$. Previous studies have suggested that zones o to 2 of the aortic arch are involved in $>40 \%$ of TEVAR cases. When the stent graft covers the carotid artery and LSCA, adverse events may occur. Coverage of LSCA is reportedly related to stroke, paraplegia, and arm claudication $[24,25]$. In our study, approximately half of the patients were zone 1 to 2 (51.3\%). We tried to preserve the left carotid artery and LSCA, performing 10 revascularizations with the chimney technique and supra-aortic arch vessel bypass. We also performed TEVAR with only partial coverage of the LSCA when patients had short proximal landing zones. We observed no significant differences in clinical outcomes between patients with landing zones 1 to 2 and 3 to 4 . Adequate revascularization of the left carotid artery and LSCA in patients with ruptured thoracic aortic disease could reduce cardiovascular events.

We also evaluated the prognostic factors associated with the composite outcome. We found that a low MAP was the only independent predictor of the composite outcome. Among the four patients with a MAP $<60 \mathrm{mmHg}$ on admission, three died in the hospital and one developed secondary endoleak. Similar to our findings, Echeverria et al. [26] suggested that $>4$ units of packed red blood cells in the first 24 hours, MAP < $60 \mathrm{mmHg}$ on admission, and a fresh frozen plasma to packed red blood cell ratio < 1:1.5 were independently associated with death. Our study also showed that packed red blood cell transfusion and low MAP due to aortic rupture were associated with poor clinical outcome.

We employed either two perclose devices or surgical cutdown to access the femoral artery. No significant differences in clinical outcomes were observed between 
the two access methods. Nelson et al.'s [27] randomized study comparing these two access methods for TEVAR also showed no differences in clinical outcomes.

Our study has some limitations. First, it was a single-center retrospective study, with a small sample size. This made it difficult to discern differences in clinical outcomes among the different aortic pathologies. Second, conducting a randomized study between TEVAR and open surgery is difficult because the clinical conditions, lesion location, and pathology of patients with ruptured thoracic aortic disease are variable. Hence, directly analyzing the clinical outcomes of TEVAR compared to open surgery was difficult. However, our study showed acceptable clinical outcomes compared with previous studies [3-11].

In conclusion, endovascular treatment of ruptured thoracic aortic disease showed high technical success rates and acceptable clinical outcomes. An initial low blood pressure, especially MAP ( $\leq 60 \mathrm{mmHg}$ ) was the most important predictor of clinical outcome and a high transfusion requirement in the first 24 hours was associated with poor clinical outcome. Endovascular management of ruptured thoracic aortic disease could be an alternative to surgical treatment.

\section{KEY MESSAGE}

1. Low mean arterial pressure $(\leq 60 \mathrm{mmHg})$ was found to be a significant predictor of the composite outcome and a high transfusion requirement ( $\geq 5$ units) in the first 24 hours was associated with the poor clinical outcome.

2. No significant differences in clinical outcomes between patients with landing zones 1 to 2 and 3 to 4 were observed. Adequate revascularization of the left carotid artery and left subclavian artery in patients with ruptured thoracic aortic disease could reduce cardiovascular events.

3. Endovascular treatment of ruptured thoracic aortic disease showed high technical success rates and good clinical outcomes.

\section{Conflict of interest}

No potential conflict of interest relevant to this article was reported.

\section{REFERENCES}

1. Johansson G, Markstrom U, Swedenborg J. Ruptured thoracic aortic aneurysms: a study of incidence and mortality rates. J Vasc Surg 1995;21:985-988.

2. Jonker FH, Verhagen HJ, Lin PH, et al. Open surgery versus endovascular repair of ruptured thoracic aortic aneurysms. J Vasc Surg 2011;53:1210-1216.

3. Buz S, Zipfel B, Mulahasanovic S, Pasic M, Weng Y, Hetzer R. Conventional surgical repair and endovascular treatment of acute traumatic aortic rupture. Eur J Cardiothorac Surg 2008;33:143-149.

4. Xenos ES, Minion DJ, Davenport DL, et al. Endovascular versus open repair for descending thoracic aortic rupture: institutional experience and meta-analysis. Eur J Cardiothorac Surg 2009;35:282-286.

5. Scheinert D, Krankenberg H, Schmidt A, et al. Endoluminal stent-graft placement for acute rupture of the descending thoracic aorta. Eur Heart J 2004;25:694-700.

6. Minami T, Imoto K, Uchida K, et al. Clinical outcomes of emergency surgery for acute type B aortic dissection with rupture. Eur J Cardiothorac Surg 2013;44:360-365.

7. Schermerhorn ML, Giles KA, Hamdan AD, Dalhberg SE, Hagberg R, Pomposelli F. Population-based outcomes of open descending thoracic aortic aneurysm repair. J Vasc Surg 2008;48:821-827.

8. Jonker FH, Trimarchi S, Verhagen HJ, Moll FL, Sumpio BE, Muhs BE. Meta-analysis of open versus endovascular repair for ruptured descending thoracic aortic aneurysm. J Vasc Surg 2010;51:1026-1032.

9. Fattori R, Tsai TT, Myrmel T, et al. Complicated acute type B dissection: is surgery still the best option?: a report from the International Registry of Acute Aortic Dissection. JACC Cardiovasc Interv 2008;1:395-402.

10. Leurs LJ, Harris PL, Buth J; EUROSTAR Collaborators. Secondary interventions after elective endovascular repair of degenerative thoracic aortic aneurysms: results of the European collaborators registry (EUROSTAR). J Vasc Interv Radiol 2007;18:491-495.

11. Khoynezhad A, Donayre CE, Azizzadeh A, White R; RESCUE investigators. One-year results of thoracic endovascular aortic repair for blunt thoracic aortic injury (RESCUE trial). J Thorac Cardiovasc Surg 2015;149:155-161.

12. Jonker FH, Giacovelli JK, Muhs BE, Sosa JA, Indes JE. Trends and outcomes of endovascular and open treatment for traumatic thoracic aortic injury. J Vasc Surg 
2010;51:565-571.

13. Demetriades D, Velmahos GC, Scalea TM, et al. Operative repair or endovascular stent graft in blunt traumatic thoracic aortic injuries: results of an American Association for the Surgery of Trauma Multicenter Study. J Trauma 2008;64:561-571.

14. Svensson LG, Kouchoukos NT, Miller DC, et al. Expert consensus document on the treatment of descending thoracic aortic disease using endovascular stent-grafts. Ann Thorac Surg 2008;85(1 Suppl):S1-S41.

15. Wiedemann D, Ehrlich M, Amabile P, et al. Emergency endovascular stent grafting in acute complicated type B dissection. J Vasc Surg 2014;60:1204-1208.

16. Fanelli F, Cannavale A, O'Sullivan GJ, et al. Endovascular repair of acute and chronic aortic type $\mathrm{B}$ dissections: main factors affecting aortic remodeling and clinical outcome. JACC Cardiovasc Interv 2016;9:183-191.

17. Faure EM, Canaud L, Marty-Ane C, Becquemin JP, Alric P. Endovascular management of rupture in acute type B aortic dissections. Eur J Vasc Endovasc Surg 2015;49:655660.

18. Etezadi V, Schiro B, Pena CS, Kovacs M, Benenati JF, Katzen BT. Endovascular treatment of descending thoracic aortic disease: single-center, 15-year experience. J Vasc Interv Radiol 2012;23:468-475.

19. Acher C, Acher CW, Marks E, Wynn M. Intraoperative neuroprotective interventions prevent spinal cord ischemia and injury in thoracic endovascular aortic repair. J Vasc Surg 2016;63:1458-1465.

20. Ullery BW, Cheung AT, Fairman RM, et al. Risk factors, outcomes, and clinical manifestations of spinal cord ischemia following thoracic endovascular aortic repair. J Vasc Surg 2011;54:677-684.

21. Yingbin J, Jiefei M, Jian L, et al. Evaluation of the thoracic aortic dissection treated by endografts covering a longer distance of aorta according to the location of the Adamkiewicz artery. Thorac Cardiovasc Surg 2013;61:569-574.

22. Lavingia KS, Ahanchi SS, Redlinger RE, Udgiri NR, Panneton JM. Aortic remodeling after thoracic endovascular aortic repair for intramural hematoma. J Vasc Surg 2014;60:929-936.

23. Harris KM, Braverman AC, Eagle KA, et al. Acute aortic intramural hematoma: an analysis from the International Registry of Acute Aortic Dissection. Circulation 2012;126(11 Suppl 1):S91-S96.

24. Feezor RJ, Martin TD, Hess PJ, et al. Risk factors for perioperative stroke during thoracic endovascular aortic repairs (TEVAR). J Endovasc Ther 2007;14:568-573.

25. Peterson BG, Eskandari MK, Gleason TG, Morasch MD. Utility of left subclavian artery revascularization in association with endoluminal repair of acute and chronic thoracic aortic pathology. J Vasc Surg 2006;43:433-439.

26. Echeverria AB, Branco BC, Goshima KR, Hughes JD, Mills JL Sr. Outcomes of endovascular management of acute thoracic aortic emergencies in an academic level 1 trauma center. Am J Surg 2014;208:974-980.

27. Nelson PR, Kracjer Z, Kansal N, et al. A multicenter, randomized, controlled trial of totally percutaneous access versus open femoral exposure for endovascular aortic aneurysm repair (the PEVAR trial). J Vasc Surg 2014;59:11811193. 